\title{
The Effect of Type 2 Diabetes Mellitus on the
} Short-Term Outcomes and Prognosis of Stage I-III Colorectal Cancer: A Propensity Score Matching Analysis

\author{
Yong Cheng' \\ Yu-Xi Cheng iD \\ Xiao-Yu Liu \\ Bing Kang (D) ${ }^{2}$ \\ Wei Tao (D) \\ Dong Peng (D) \\ 'Department of Gastrointestinal Surgery, \\ The First Affiliated Hospital of Chongqing \\ Medical University, Chongqing, 4000I6, \\ People's Republic of China; ${ }^{2}$ Department \\ of Clinical Nutrition, The First Affiliated \\ Hospital of Chongqing Medical University, \\ Chongqing, 400016, People's Republic of \\ China
}

Purpose: The purpose of the current study was to analyze the effect of type 2 diabetes mellitus (T2DM) on the short-term outcomes and prognosis of stage I-III colorectal cancer (CRC) undergoing primary surgery.

Methods: Patients who underwent primary CRC surgery were retrospectively collected from Jan 2011 to Jan 2020 in a single clinical center. The short-term outcomes and prognosis were compared between T2DM group and non-T2DM group using propensity score matching (PSM) analysis.

Results: A total of 4250 patients were included in this study. There were 521 patients with T2DM and 3729 patients without T2DM. After 1:1 ratio PSM, there were 519 T2DM patients and 519 nonT2DM patients left in this study. No significant difference was found in baseline information after $\operatorname{PSM}(p>0.05)$. T2DM had higher overall complications $(\mathrm{p}=0.033)$ after PSM in terms of short-term outcomes. As for prognosis, T2DM group had worse overall survival (OS) in all stages ( $\mathrm{p}=0.044)$, stage I $(p=0.009)$ and stage II ( $p=0.021)$ of CRC and T2DM group had worse disease-free survival (DFS) than non-T2DM group in stage I $(\mathrm{p}=0.008)$ of CRC before PSM. However, T2DM did not affect the overall survival (OS) or disease-free survival (DFS) on different stages of CRC after PSM $(p>0.05)$. Moreover, T2DM was not an independent predictor of OS or DFS ( $p>0.05$ ).

Conclusion: T2DM increased overall complications after primary CRC surgery. However, T2DM might not affect OS and DFS of stage I-III CRC patients.

Keywords: colorectal cancer, type 2 diabetes mellitus, outcomes, prognosis

\section{Introduction}

Colorectal cancer (CRC) is the third leading cause of death and the fourth most common cancer in the world. ${ }^{1}$ More than 900,000 people die every year because of CRC. $^{2}$ In addition, the number of CRC patients is expected to increase in the future, ${ }^{3}$ and surgery is still the main treatment for CRC. ${ }^{4,5}$

Type 2 diabetes mellitus (T2DM) causes a significant burden on patients and society globally, ${ }^{6}$ and T2DM accounts for increased morbidity and mortality. ${ }^{7}$ The number of T2DM patients is expected to reach 700 million in $2045 .^{8}$

Cancer and T2DM are two global public health problems and are also regarded as the world's major lethal and disabling diseases. ${ }^{9}$ They share various common risk factors (high blood pressure, obesity, hyperlipidemia) and have a similar etiological mechanism. ${ }^{10,11}$
Correspondence: Dong Peng Department of Gastrointestinal Surgery, The First Affiliated Hospital of Chongqing Medical University, Chongqing, 400016,

People's Republic of China

Tel +862389011014

Email carry_dong@I26.com 
As previous studies reported, T2DM could increase the incidence of CRC. ${ }^{12}$ However, the effect of T2DM on CRC remains controversial. ${ }^{13,14}$ Furthermore, there have been no propensity score matching (PSM) studies concerning the relationship between CRC and T2DM. Therefore, the purpose of the current study was to analyze the effect of T2DM on the short-term outcomes and prognosis of CRC patients undergoing primary surgery using PSM analysis.

\section{Methods}

\section{Patients}

Patients who underwent primary CRC surgery were retrospectively collected from Jan 2011 to Jan 2020 in a single clinical center. This study was conducted in accordance with the World Medical Association Declaration of Helsinki. Ethical approval from the First Affiliated Hospital of Chongqing Medical University board was obtained (2021-336) and all the patients signed informed consent.

\section{Inclusion and Exclusion Criteria}

We included the patients who underwent primary CRC surgery and the pathology diagnosed CRC from a database of a single clinical center $(\mathrm{n}=5473)$. The exclusion criteria were as follows: 1) stage IV CRC ( $\mathrm{n}=875) ; 2)$ incomplete medical records $(\mathrm{n}=323)$; and 3$)$ non-R0 resection $(n=25)$. Finally, a total of 4250 patients were included in the current study.

\section{Surgery Management}

All patients underwent radical resection according to the clinical guideline and total mesorectal excision or complete mesocolic excision was performed, and the pathology confirmed R0 resection. Patients were regularly followed up every 3 months for the first 3 years and every 6 months for the following 2 years.

\section{Definitions}

The tumor node metastasis (TNM) stage was diagnosed according to the AJCC eighth edition. ${ }^{15}$ Overall survival (OS) was defined as the time from primary CRC surgery to the last follow-up (when patients were alive) or death. Disease-free survival (DFS) was defined as the time from primary CRC surgery to recurrence, death or last follow-up (when patients were alive). The complications were defined according to the Clavien-Dindo classification, ${ }^{16}$ and major complications were defined as $\geq$ III classification complications.

\section{Data Collection}

Patient information was retrospectively collected from inpatient and outpatient system, and the follow-up information was collected from the outpatient system and telephone interviews. The peri-operative information included age, sex, body mass index (BMI), smoking, drinking, family history, hypertension, coronary heart disease (CHD), adjuvant therapy, tumor stage and tumor location. The short-term outcomes included operation time, blood loss, retrieved lymph nodes, hospital stay, overall complications, major complications, postoperative 30-day death, reoperation and anastomotic leakage. The prognosis included OS and DFS.

\section{PSM}

To minimize the bias of baseline information, PSM was conducted between T2DM group and non-T2DM group. Nearest neighbor matching was performed without replacement at a 1:1 ratio and a caliper width with a 0.01 standard deviation was specified. The baseline information was matched including age, sex, BMI, smoking, drinking, family history, hypertension, CHD, adjuvant therapy, tumor location and tumor stage.

\section{Statistical Analysis}

Continuous variables are expressed as the mean $\pm \mathrm{SD}$, and independent-sample $t$-test was used to compare the difference between T2DM group and non-T2DM group. Frequency variables are expressed as $\mathrm{n}(\%)$, and Chisquare test or Fisher's exact test was used. The KaplanMeier curve was conducted to compare T2DM on different pathological stages, and Cox regression analyses were performed to identify independent predictive factors for OS and DFS. Data were analyzed using SPSS (version 22.0) statistical software. A bilateral $\mathrm{p}$ value $<0.05$ was considered statistically significant.

\section{Results \\ Patients}

A total of 4250 patients were included in this study according to the inclusion and exclusion criteria. There were 521 patients with T2DM and 3729 patients without T2DM. After 1:1 ratio PSM, there were 519 T2DM patients and 519 non- 
T2DM patients left in this study. The flow chart of inclusion, exclusion and PSM is shown in Figure 1.

\section{Baseline Information}

Baseline information was compared between T2DM and non-T2DM groups, and T2DM group had an older age $(\mathrm{p}=0.000)$, higher BMI $(\mathrm{p}=0.000)$, higher portion of hypertension $(\mathrm{p}=0.000)$, higher portion of CHD $(\mathrm{p}=0.000)$ and lower portion of adjuvant therapy $(\mathrm{p}=0.011)$ before PSM. However, no significant difference was found after PSM in terms of age, sex, BMI, smoking, drinking, family history, hypertension, CHD, adjuvant therapy, tumor location or tumor stage $(p>0.05)$ (Table 1).

\section{Short-Term Outcomes}

The short-term outcomes including operation time, blood loss, retrieved lymph nodes, hospital stay, overall complications, major complications, postoperative 30-day death, reoperation and anastomotic leakage were compared between T2DM and non-T2DM group. T2DM group had longer hospital stay $(\mathrm{p}=0.002)$, higher overall complications $(\mathrm{p}=0.000)$ and higher anastomotic leakage $(\mathrm{p}=0.022)$ before PSM. After PSM, T2DM had higher overall complications $(\mathrm{p}=0.033)$ (Table 2$)$.

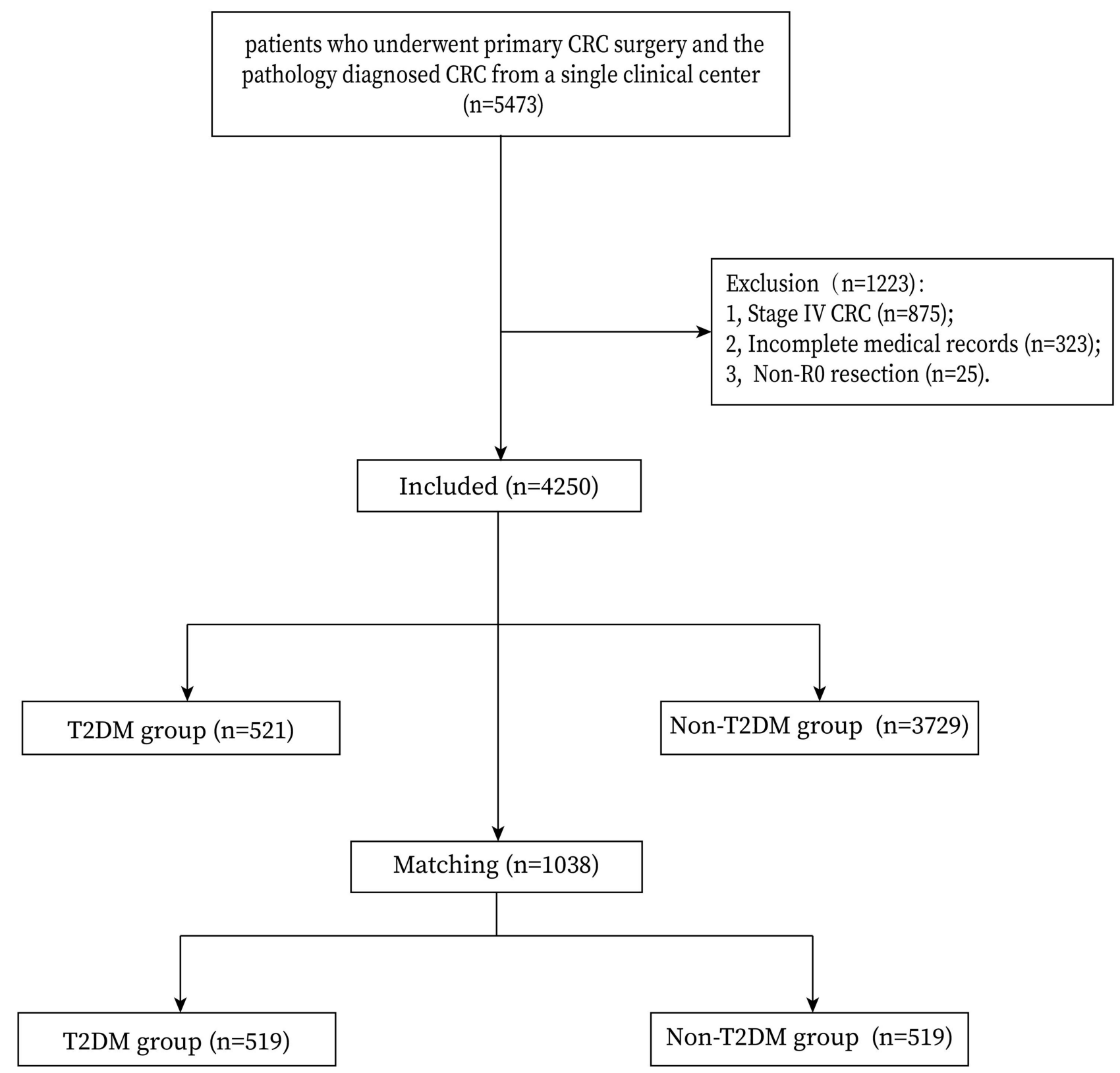

Figure I Flow chart of patient selection. 
Table I Baseline Characteristics Before and After PSM

\begin{tabular}{|c|c|c|c|c|c|c|}
\hline \multirow[t]{2}{*}{ Characteristics } & \multicolumn{3}{|c|}{ Before PSM } & \multicolumn{3}{|c|}{ After PSM } \\
\hline & T2DM (52I) & Non-T2DM (3729) & P value & T2DM (5 I9) & Non-T2DM (5 I9) & $P$ value \\
\hline Age (years) & $68.2 \pm 9.7$ & $62.2 \pm 12.3$ & $0.000 *$ & $68.2 \pm 9.7$ & $67.9 \pm 11.1$ & 0.662 \\
\hline Sex & & & 0.448 & & & 0.572 \\
\hline Male & 298 (57.2\%) & 2198 (58.9\%) & & $296(57.0 \%)$ & 305 (58.8\%) & \\
\hline Female & $223(42.8 \%)$ & $|53|$ (4I.I\%) & & $223(43.0 \%)$ & $214(41.2 \%)$ & \\
\hline BMI $\left(\mathrm{kg} / \mathrm{m}^{2}\right)$ & $23.6 \pm 3.3$ & $22.6 \pm 3.2$ & $0.000 *$ & $23.5 \pm 3.3$ & $23.4 \pm 3.3$ & 0.485 \\
\hline Smoking & I8I (34.7\%) & 1426 (38.2\%) & 0.123 & 180 (34.7\%) & $18 \mid(34.9 \%)$ & 0.948 \\
\hline Drinking & I5I (29.0\%) & II 50 (30.8\%) & 0.398 & 150 (28.9\%) & $148(28.5 \%)$ & 0.891 \\
\hline Family history & $18(3.5 \%)$ & 109 (2.9\%) & 0.504 & $18(3.5 \%)$ & $17(3.3 \%)$ & 0.863 \\
\hline Hypertension & $296(56.8 \%)$ & $812(21.8 \%)$ & $0.000 *$ & 294 (56.6\%) & 309 (59.5\%) & 0.345 \\
\hline CHD & $56(10.7 \%)$ & $123(3.3 \%)$ & $0.000 *$ & 54 (10.4\%) & $52(10.0 \%)$ & 0.838 \\
\hline Tumor location & & & 0.325 & & & 0.495 \\
\hline Colon & $25 \mathrm{I}(48.2 \%)$ & $|7| \mid(45.9 \%)$ & & 250 (48.2\%) & $26 \mid(50.3 \%)$ & \\
\hline Rectum & $270(51.8 \%)$ & 2018 (54.1\%) & & $269(51.8 \%)$ & $258(49.7 \%)$ & \\
\hline Adjuvant therapy & $4 \mathrm{I}(7.9 \%)$ & $192(5.1 \%)$ & $0.011 *$ & 40 (7.7\%) & $32(6.2 \%)$ & 0.499 \\
\hline Tumor stage & & & 0.482 & & & 0.894 \\
\hline I & $96(18.4 \%)$ & 754 (20.2\%) & & $96(18.5 \%)$ & 102 (19.7\%) & \\
\hline II & 222 (42.6\%) & 1610 (43.2\%) & & 220 (42.4\%) & $217(41.8 \%)$ & \\
\hline III & 203 (39.0\%) & 1365 (36.6\%) & & $203(39.1 \%)$ & $200(38.5 \%)$ & \\
\hline
\end{tabular}

Notes: Variables are expressed as the mean \pm SD, $n(\%)$, *P-value $<0.05$.

Abbreviations: T2DM, type 2 diabetes mellitus; BMI, body mass index; PSM, propensity score matching; CHD, coronary heart disease; PSM, propensity score matching.

Table 2 Short-Term Outcomes Before and After PSM

\begin{tabular}{|l|l|l|l|l|l|l|}
\hline \multirow{2}{*}{ Characteristics } & \multicolumn{3}{|c|}{ Before PSM } & \multicolumn{3}{c|}{ After PSM } \\
\cline { 2 - 7 } & T2DM (52I) & Non-T2DM (3729) & P value & T2DM (5 I9) & Non-T2DM (5 19) & P value \\
\hline Operation time (min) & $225.1 \pm 77.5$ & $222.4 \pm 76.1$ & 0.463 & $224.6 \pm 77.3$ & $227.0 \pm 82.1$ & 0.640 \\
Blood loss (mL) & $97.4 \pm 107.6$ & $100.8 \pm 128.0$ & 0.560 & $95.6 \pm 103.0$ & $104.7 \pm 143.1$ & 0.240 \\
Retrieved lymph nodes & $14.6 \pm 9.3$ & $14.7 \pm 7.2$ & 0.793 & $14.7 \pm 9.3$ & $14.2 \pm 7.0$ & 0.389 \\
Hospital stay (days) & $12.5 \pm 11.2$ & $11.2 \pm 8.4$ & $0.002 *$ & $12.5 \pm 11.2$ & $11.6 \pm 7.7$ & 0.139 \\
Overall complications & $149(28.6 \%)$ & $778(20.9 \%)$ & $0.000 *$ & $148(28.5 \%)$ & $118(22.7 \%)$ & 0.033 \\
Major complications & $17(3.3 \%)$ & $82(2.2 \%)$ & 0.132 & $17(3.3 \%)$ & $10(1.9 \%)$ & 0.172 \\
Postoperative 30-day death & $3(0.6 \%)$ & $9(0.2 \%)$ & 0.174 & $3(0.6 \%)$ & $0(0.0 \%)$ & 0.249 \\
Re-operation & $12(2.3 \%)$ & $59(1.6 \%)$ & 0.229 & $12(2.3 \%)$ & $10(1.9 \%)$ & 0.666 \\
Anastomotic leakage & $20(3.8 \%)$ & $82(2.2 \%)$ & $0.022^{*}$ & $20(3.8 \%)$ & $10(1.9 \%)$ & 0.064 \\
\hline
\end{tabular}

Notes: Variables are expressed as the mean \pm SD, n (\%), *P-value $<0.05$.

Abbreviations: T2DM, type 2 diabetes mellitus; PSM, propensity score matching.

\section{Univariate and Multivariate Analyses of OS}

The median follow-up time was 37 (1-114) months, univariate and multivariate analyses were conducted to find the predictors for OS of the 1038 patients after PSM. In univariate analysis, age $(\mathrm{p}=0.000, \mathrm{HR}=2.007,95 \% \mathrm{CI}=1.486-2.710)$, BMI $(\mathrm{p}=0.008, \mathrm{HR}=0.658,95 \% \mathrm{CI}=0.484-0.895)$, tumor stage $(p=0.000, H R=1.556,95 \% C I=1.253-1.931)$, overall complications $(\mathrm{p}=0.000, \mathrm{HR}=1.898,95 \% \mathrm{CI}=1.407-2.561)$, major complications $(\mathrm{p}=0.000, \mathrm{HR}=2.940,95 \% \mathrm{CI}=1.635-$
5.287) and anastomotic leakage $(\mathrm{p}=0.007, \mathrm{HR}=2.542,95 \%$ $\mathrm{CI}=1.298-4.979)$ were predictors; however, T2DM was not a predictor $(\mathrm{p}=0.764, \mathrm{HR}=0.956,95 \% \mathrm{CI}=0.712-1.283)$. In multivariate analysis, age $(\mathrm{p}=0.000, \mathrm{HR}=1.843, \quad 95 \%$ $\mathrm{CI}=1.350-2.515), \quad \mathrm{BMI} \quad(\mathrm{p}=0.029, \quad \mathrm{HR}=0.706, \quad 95 \%$ $\mathrm{CI}=0.516-0.965)$, tumor stage $(\mathrm{p}=0.000, \mathrm{HR}=1.587,95 \%$ $\mathrm{CI}=1.273-1.979), \quad$ overall complications $\quad(\mathrm{p}=0.016$, $\mathrm{HR}=1.496,95 \% \mathrm{CI}=1.077-2.077)$ and major complications $(\mathrm{p}=0.010, \mathrm{HR}=2.767,95 \% \mathrm{CI}=1.270-6.029)$ were independent predictors for OS (Table 3). 
Table 3 Univariate and Multivariate Analyses of Overall Survival (After PSM)

\begin{tabular}{|c|c|c|c|c|}
\hline \multirow[t]{2}{*}{ Risk Factors } & \multicolumn{2}{|c|}{ Univariate Analysis } & \multicolumn{2}{|c|}{ Multivariate Analysis } \\
\hline & HR (95\% Cl) & P value & HR (95\% Cl) & $P$ value \\
\hline Age (>I $1 \leq 69$ years) & $2.007(1.486-2.710)$ & $0.000 *$ & I.843 (I.350-2.5I5) & $0.000 *$ \\
\hline Sex (male/female) & 0.891 (0.660-I.202) & 0.449 & & \\
\hline $\operatorname{BMI}\left(>/ \leq 23.4 \mathrm{~kg} / \mathrm{m}^{2}\right)$ & $0.658(0.484-0.895)$ & $0.008^{*}$ & $0.706(0.516-0.965)$ & $0.029 *$ \\
\hline Hypertension (yes/no) & $1.032(0.767-1.390)$ & 0.834 & & \\
\hline T2DM (yes/no) & $0.956(0.7 \mid 2-1.283)$ & 0.764 & & \\
\hline Tumor site (colon/rectum) & I.I93 (0.889-I.60I) & 0.240 & & \\
\hline Tumor stage (III/II/I) & $1.556(1.253-1.931)$ & $0.000 *$ & I.587 (I.273-I.979) & $0.000^{*}$ \\
\hline Smoking (yes/no) & $0.884(0.646-1.208)$ & 0.438 & & \\
\hline Drinking (yes/no) & $0.775(0.55 \mathrm{I}-1.089)$ & 0.142 & & \\
\hline Family history (yes/no) & $0.863(0.382-1.948)$ & 0.723 & & \\
\hline CHD (yes/no) & $1.086(0.658-1.79 \mid)$ & 0.747 & & \\
\hline Adjuvant therapy (yes/no) & $0.799(0.354-1.806)$ & 0.590 & & \\
\hline Overall complications (yes/no) & $\mathrm{I} .898(\mathrm{I} .407-2.56 \mathrm{I})$ & $0.000 *$ & $1.496(1.077-2.077)$ & $0.016 *$ \\
\hline Major complications (yes/no) & $2.940(1.635-5.287)$ & $0.000 *$ & $2.767(1.270-6.029)$ & $0.010^{*}$ \\
\hline Anastomotic leakage (yes/no) & $2.542(1.298-4.979)$ & $0.007 *$ & $1.225(0.5 \mid 3-2.929)$ & 0.648 \\
\hline
\end{tabular}

Note: *P-value $<0.05$.

Abbreviations: HR, hazard ratio; Cl, confidence interval; BMI, body mass index; T2DM, type 2 diabetes mellitus; CHD, coronary heart disease; PSM, propensity score matching.

\section{Univariate and Multivariate Analyses of DFS}

As for DFS, in univariate analysis, age $(\mathrm{p}=0.000, \mathrm{HR}=1.747$, 95\% CI $=1.325-2.303)$, BMI $(\mathrm{p}=0.019, \mathrm{HR}=0.714,95 \%$ $\mathrm{CI}=0.538-0.947)$, tumor stage $(\mathrm{p}=0.000, \mathrm{HR}=1.796,95 \%$ $\mathrm{CI}=1.447-2.229), \quad$ overall $\quad$ complications $\quad(\mathrm{p}=0.001$,
$\mathrm{HR}=1.622,95 \% \mathrm{CI}=1.221-2.155)$, major complications $(\mathrm{p}=0.002, \mathrm{HR}=2.506,95 \% \mathrm{CI}=1.398-4.494)$ and anastomotic leakage $(\mathrm{p}=0.025, \mathrm{HR}=2.154,95 \% \mathrm{CI}=1.103-4.208)$ were predictors; however, T2DM was not a predictor $(\mathrm{p}=0.353$, $\mathrm{HR}=0.878,95 \% \mathrm{CI}=0.667-1.155)$. In multivariate analysis, age $(\mathrm{p}=0.001, \mathrm{HR}=1.656,95 \% \mathrm{CI}=1.245-2.204)$, tumor

Table 4 Univariate and Multivariate Analyses of Disease-Free Survival (After PSM)

\begin{tabular}{|c|c|c|c|c|}
\hline \multirow[t]{2}{*}{ Risk Factors } & \multicolumn{2}{|c|}{ Univariate Analysis } & \multicolumn{2}{|c|}{ Multivariate Analysis } \\
\hline & HR (95\% Cl) & $P$ value & HR $(95 \% \mathrm{CI})$ & $P$ value \\
\hline Age $(>1 \leq 69$ years $)$ & 1.747 (I.325-2.303) & $0.000 *$ & $1.656(1.245-2.204)$ & $0.00 I^{*}$ \\
\hline Sex (male/female) & $0.894(0.676-1.181)$ & 0.430 & & \\
\hline $\mathrm{BMI}\left(>1 \leq 23.4 \mathrm{~kg} / \mathrm{m}^{2}\right)$ & $0.714(0.538-0.947)$ & $0.019 *$ & $0.754(0.566-1.004)$ & 0.053 \\
\hline Hypertension (yes/no) & $1.039(0.788-1.370)$ & 0.786 & & \\
\hline T2DM (yes/no) & $0.878(0.667-1.155)$ & 0.353 & & \\
\hline Tumor site (colon/ rectum) & $1.094(0.833-1.438)$ & 0.518 & & \\
\hline Tumor stage $(I I I / I I / I)$ & 1.796 (I.447-2.229) & $0.000 *$ & $1.54 \mid(1.258-1.888)$ & $0.000 *$ \\
\hline Smoking (yes/no) & $0.836(0.623-1.121)$ & 0.230 & & \\
\hline Drinking (yes/no) & $0.777(0.566-1.066)$ & 0.118 & & \\
\hline Family history (yes/no) & $0.737(0.327-1.660)$ & 0.461 & & \\
\hline CHD (yes/no) & 1.011 (0.63I-I.622) & 0.962 & & \\
\hline Adjuvant therapy (yes/no) & $0.996(0.510-1.945)$ & 0.991 & & \\
\hline Overall complications (yes/no) & $1.622(1.221-2.155)$ & $0.001 *$ & $1.325(0.97 \mid-1.809)$ & 0.076 \\
\hline Major complications (yes/no) & $2.506(1.398-4.494)$ & $0.002^{*}$ & $2.55 I(I .198-5.431)$ & $0.015^{*}$ \\
\hline Anastomotic leakage (yes/no) & $2.154(1.103-4.208)$ & $0.025^{*}$ & $1.217(0.522-2.837)$ & 0.649 \\
\hline
\end{tabular}

Note: *P-value $<0.05$.

Abbreviations: HR, hazard ratio; Cl, confidence interval; BMI, body mass index; T2DM, type 2 diabetes mellitus; CHD, coronary heart disease; PSM, propensity score matching. 
stage $(\mathrm{p}=0.000, \mathrm{HR}=1.541,95 \% \mathrm{CI}=1.258-1.888)$ and major complications $(\mathrm{p}=0.015, \mathrm{HR}=2.551,95 \% \mathrm{CI}=1.198-5.431)$ were independent predictors for DFS (Table 4).

\section{Prognosis in Different Tumor Stages}

To analyze the effect of T2DM on prognosis on different stages of CRC, the Kaplan-Meier curve was conducted before and after PSM. Before PSM, T2DM group had worse OS in all stages $(p=0.044)$, stage $I(p=0.009)$ and stage II ( $p=0.021)$ (Figure 2$)$, and in terms of DFS, T2DM group had worse DFS than non-T2DM group in stage I ( $p=0.008)$ (Figure 3). However, there were no difference in all stages, stage I, stage II and stage III in terms of OS or DFS after PSM ( $>0.05)$ (Figures 4 and 5).

\section{OS before PSM}

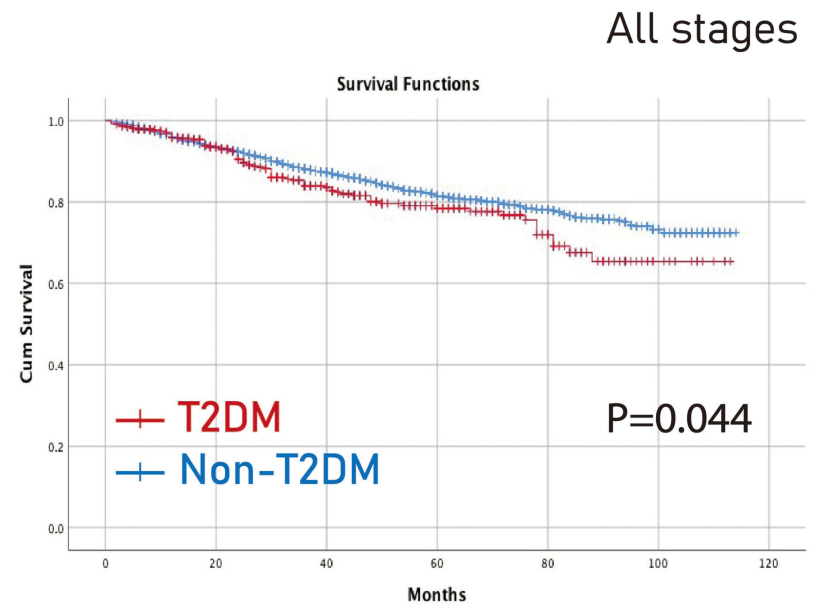

A

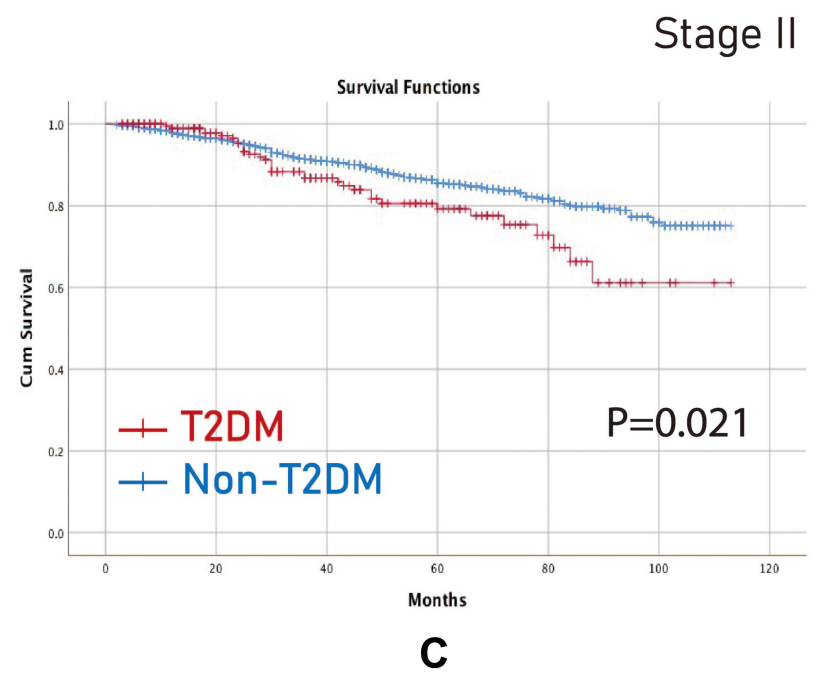

\section{Discussion}

In this study, T2DM had higher overall complications after PSM. As for prognosis, T2DM did not affect the OS or DFS on different stages of CRC after PSM, furthermore, T2DM was not an independent predictor of OS or DFS.

T2DM increased the global medical burden and economic burden, ${ }^{6,7}$ and furthermore, T2DM could increase the incidence of digestive cancers including esophageal cancer, ${ }^{17}$ gastric cancer $^{18}$ and CRC. ${ }^{12}$ However, there remained controversial about the outcomes of T2DM on CRC. Some studies reported that T2DM affected OS or DFS, ${ }^{13,14}$ a meta-analysis also reported that CRC patients with concurrent T2DM had worse DFS. ${ }^{19}$ However, other studies reported its negative influence. ${ }^{20-22}$

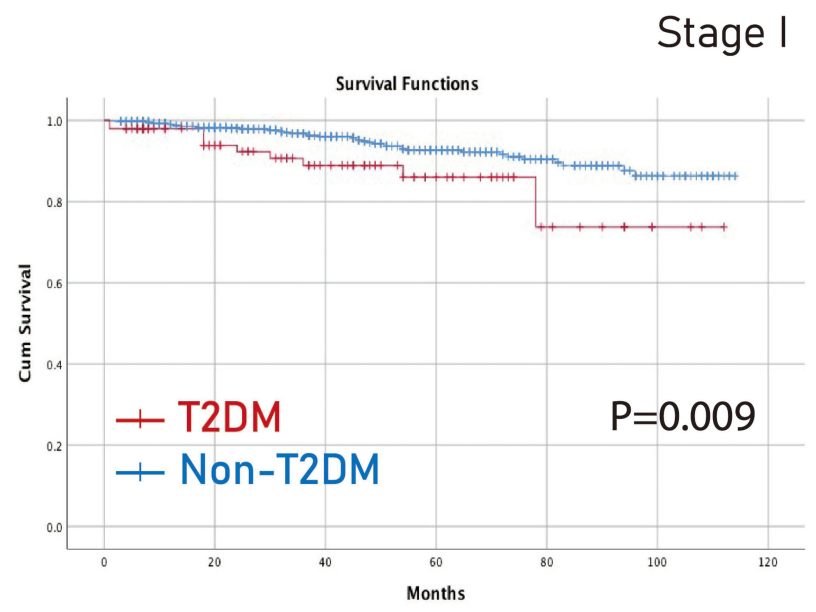

B

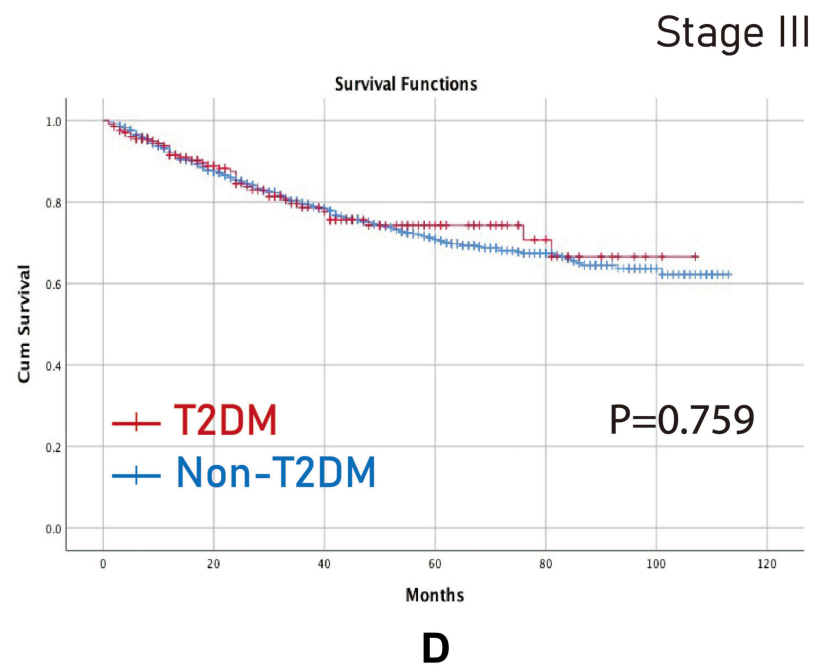

Figure 2 OS before PSM. (A) All stages; (B) Stage I; (C) Stage II; (D) Stage III.

Abbreviations: OS, overall survival; T2DM, type 2 diabetes mellitus; PSM, propensity score matching. 


\section{DFS before PSM}

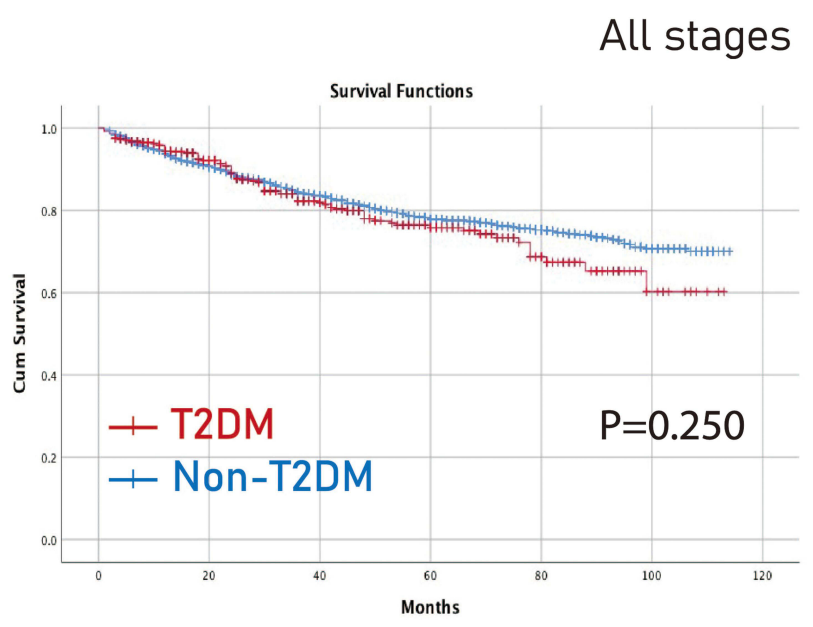

A

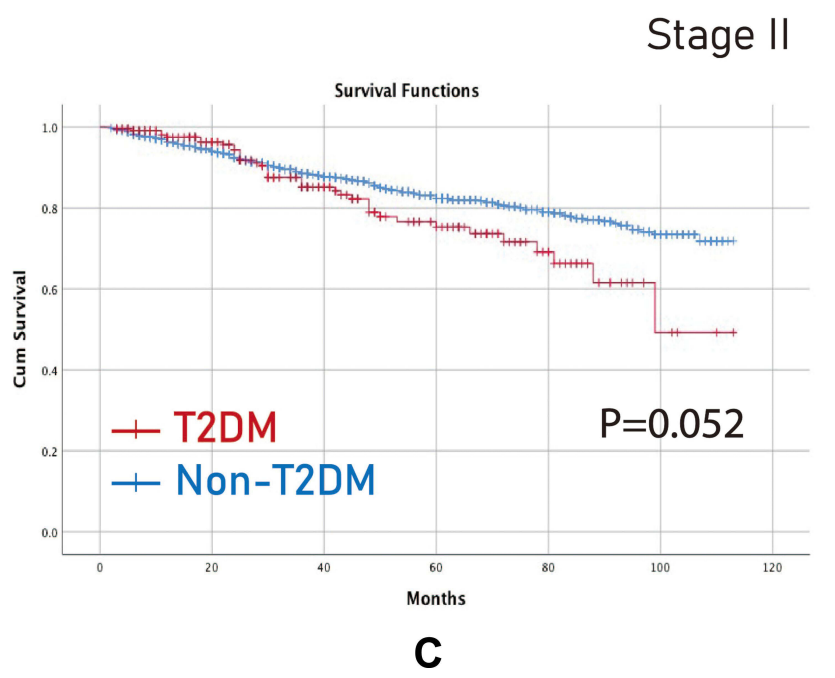

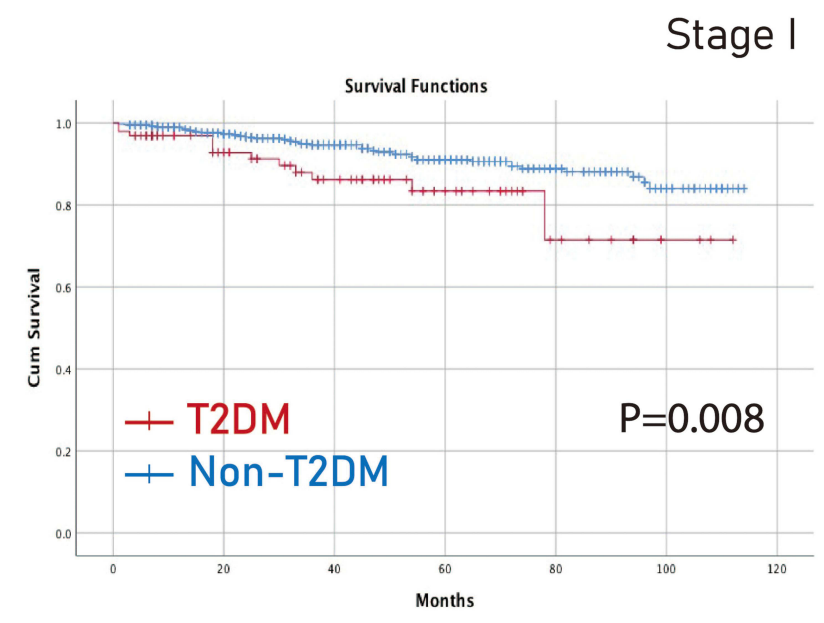

B

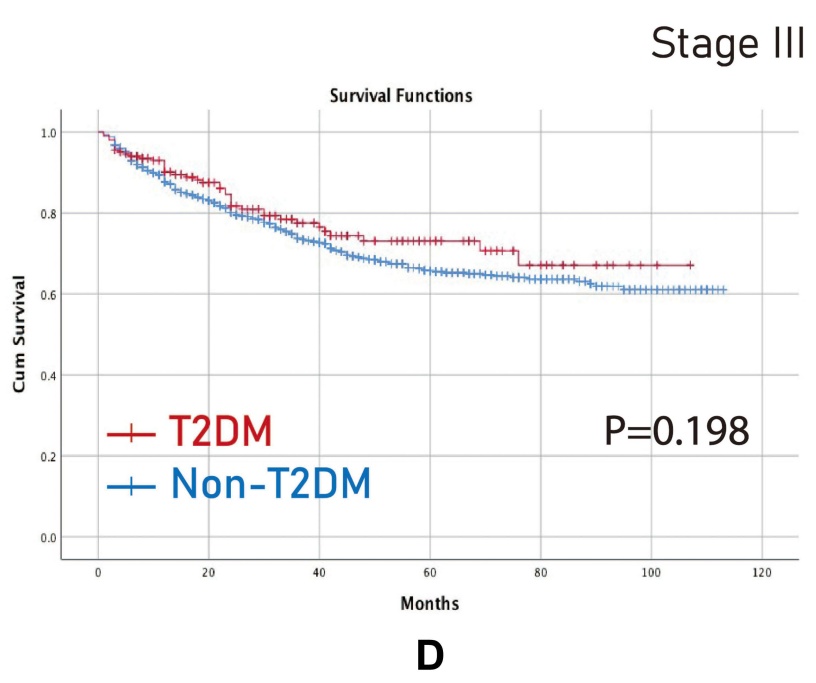

Figure 3 DFS before PSM. (A) All stages; (B) Stage I; (C) Stage II; (D) Stage III.

Abbreviations: DFS, disease-free survival; T2DM, type 2 diabetes mellitus; PSM, propensity score matching.

The mechanism of the outcomes between CRC and T2DM remained unclear, but some hypotheses have been reported. T2DM might enhance the chemo-resistance of $\mathrm{CRC}$ and hyperglycemic status affected the sensitivity to chemotherapy through multiple regulatory routes. ${ }^{23,24}$ Epidemiological studies reported metformin improved OS and DFS. Metformin enhanced response to radiotherapy and chemotherapy through synergistic interactions, indicating that it might help induce tumor regression. ${ }^{25,26}$

PSM analysis is a statistical technique, which can deal with confounding bias and mimic a randomized clinical trial, improving the level of evidence in studies. ${ }^{27}$ As far as we know, there were no related PSM studies about
T2DM and CRC, and this is the first study to analyze the potential effect of T2DM on CRC using PSM.

In this study, we analyzed the differences in surgical outcomes between T2DM group and non-T2DM group. It was found that the T2DM group had higher overall complications before and after PSM. Yap et $\mathrm{al}^{28}$ reported higher overall complications and longer hospital stay in patients with T2DM, and higher complications were reported in another study as well. ${ }^{29}$ The probable reason was that T2DM had poorer healing or comorbid with cardiovascular diseases. ${ }^{28}$ Therefore, for surgeons, adequate perioperative management should be done for patients with concurrent $\mathrm{CRC}$ and T2DM. 


\section{OS after PSM}

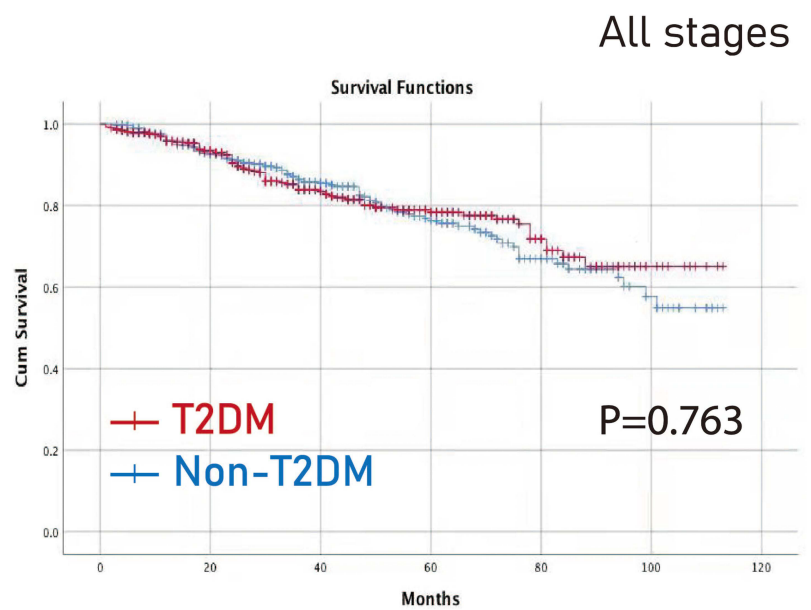

A

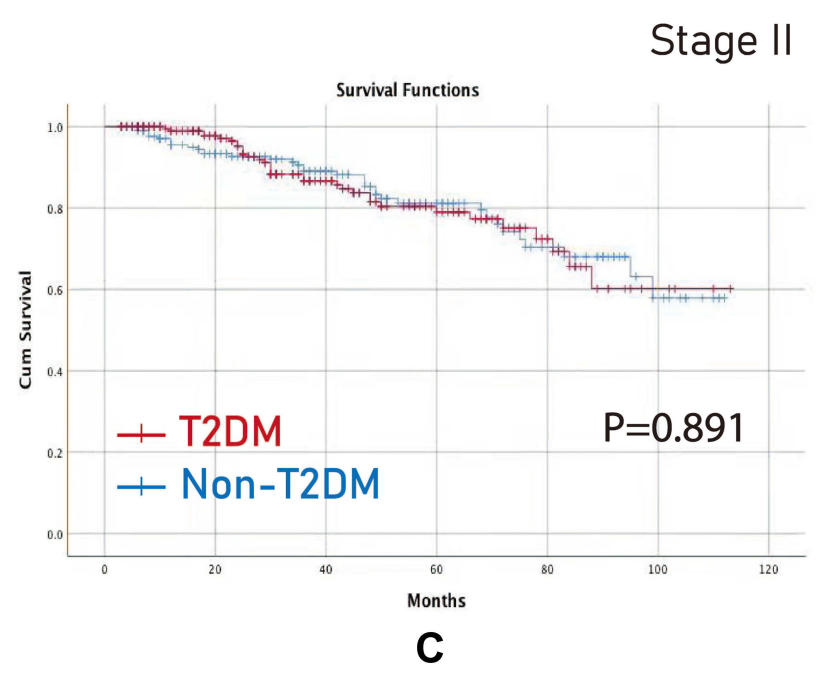

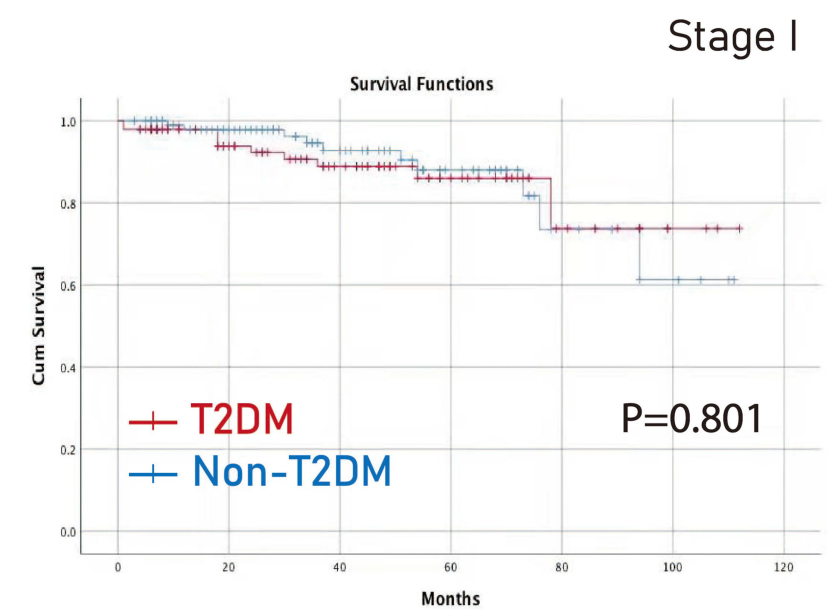

B

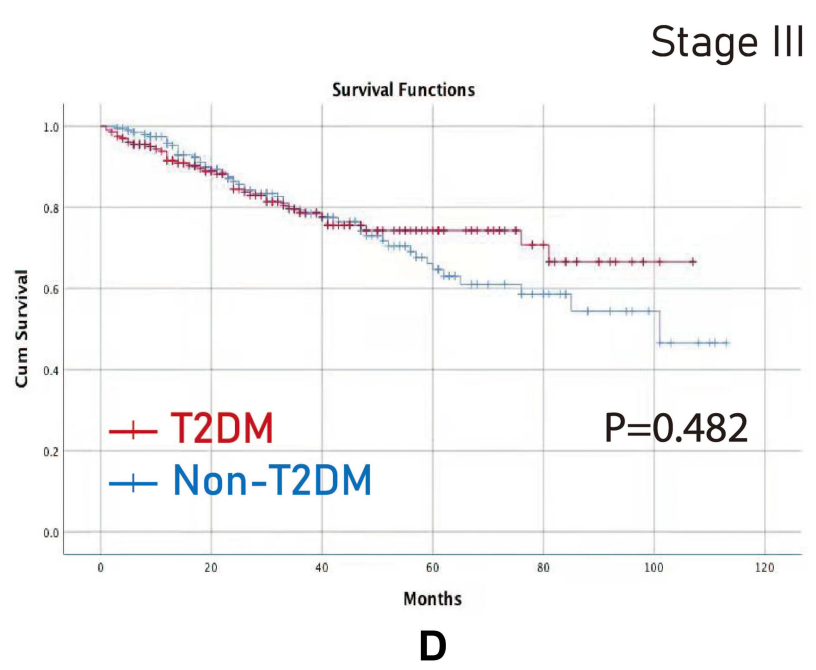

Figure 4 OS after PSM. (A) All stages; (B) Stage I; (C) Stage II; (D) Stage III.

Abbreviations: OS, overall survival; T2DM, type 2 diabetes mellitus; PSM, propensity score matching.

There were many factors that affected the prognosis of CRC, including age, tumor stage, and complications. ${ }^{30-32}$ In this study, we had the similar findings consistent with previous studies. In this study, T2DM was not a predictive factor in multivariate analysis. Furthermore, we examined T2DM at different stages of CRC to explore the exact impact of T2DM on OS and DFS. T2DM group had worse OS in all stages, stage I and stage II CRC and T2DM group had worse DFS than non-T2DM group in stage I CRC before PSM. We speculated that the possible reason was that patients had a longer OS in early tumor stage, T2DM and its related comorbidities might affect the prognosis. However, in advanced tumor stage, the tumor itself might affect the prognosis.
However, T2DM did not affect the OS or DFS at different stages of CRC after PSM, which represented that T2DM might not affect the prognosis of stage I-III CRC. Possible reasons for the null relationship between T2DM and CRC included duration and severity of T2DM, the type of T2DM medication used might be another reason. ${ }^{21}$ Further studies are needed in the future.

There were some limitations in this study. First, this was a retrospective single-center study; Second, the course and severity of T2DM were lacking, which might affect the outcome of CRC; Third, metformin use was another factor for T2DM, which needed to be analyzed in the future; Fourth, the follow-up time was relatively short. Therefore, larger sample size and multicenter prospective randomized controlled trials should be conducted in the following experiments. 


\section{DFS after PSM}

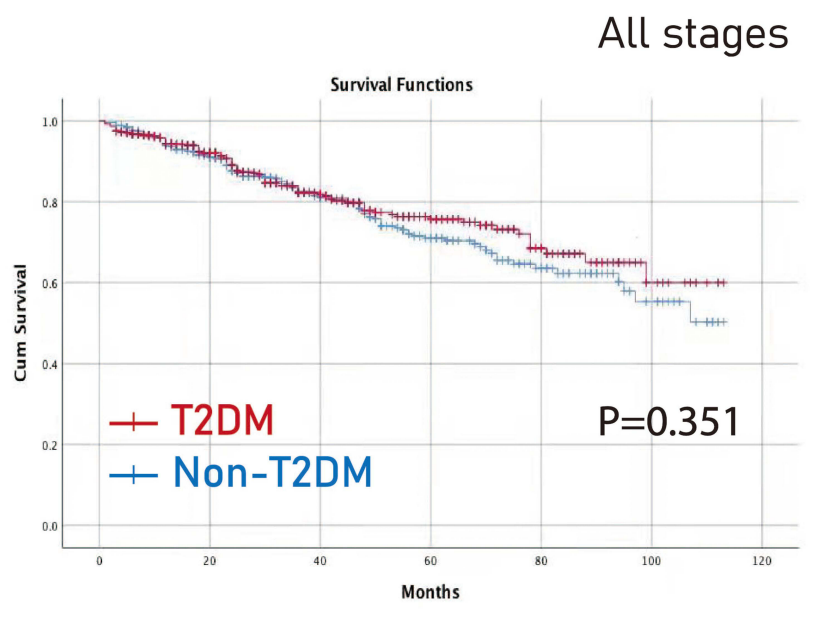

A

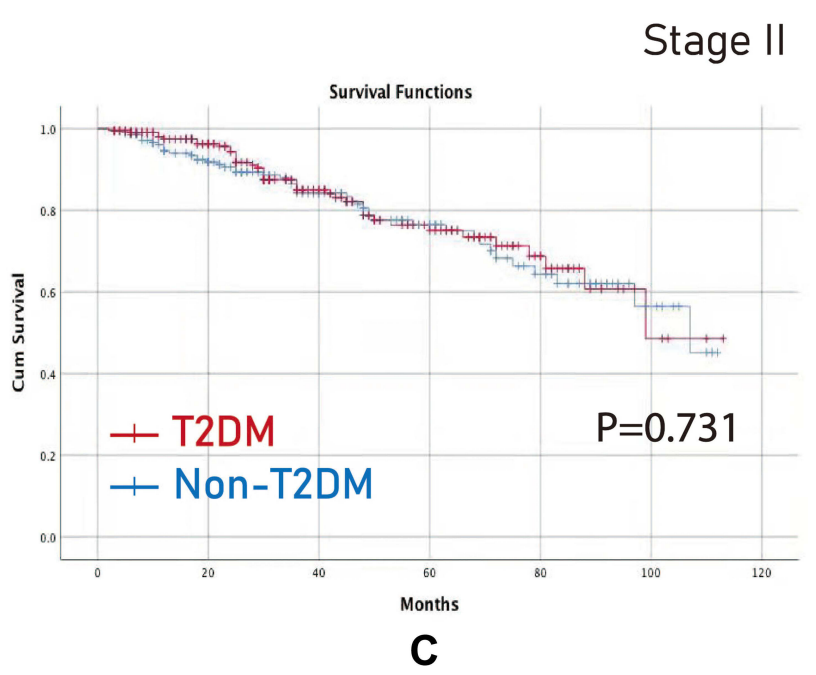

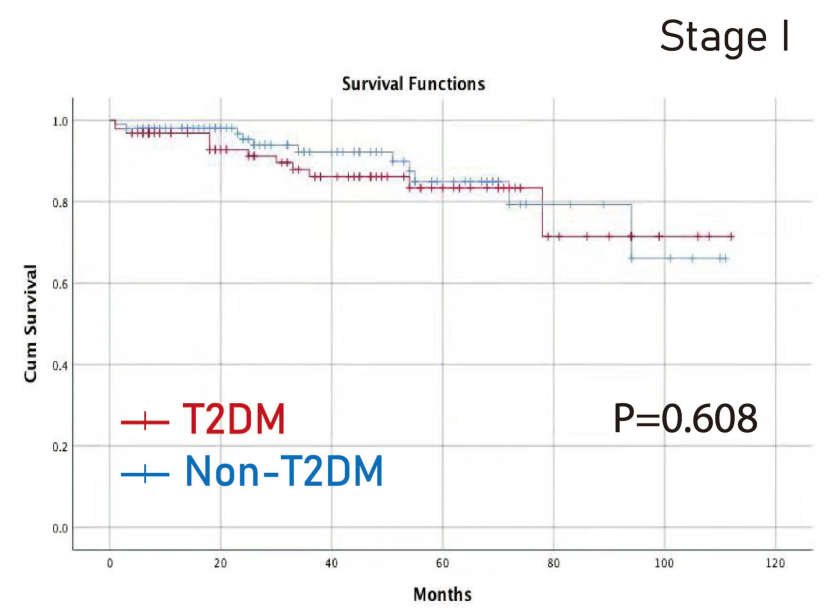

B

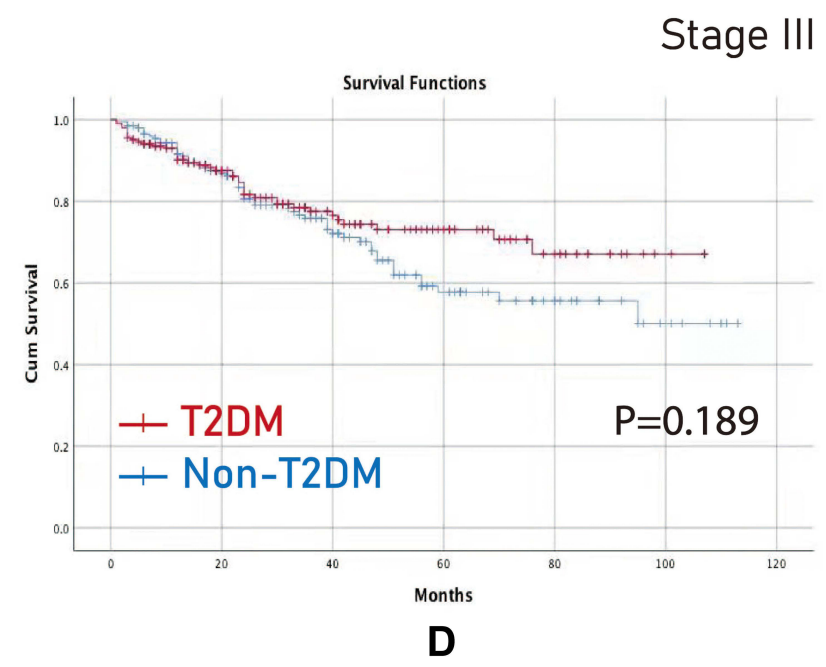

Figure 5 DFS after PSM. (A) All stages; (B) Stage I; (C) Stage II; (D) Stage III.

Abbreviations: DFS, disease-free survival; T2DM, type 2 diabetes mellitus; PSM, propensity score matching.

\section{Conclusion}

T2DM increased overall complications after primary CRC surgery. However, T2DM might not affect OS or DFS of CRC patients. Furthermore, T2DM did not affect the prognosis at different stages of CRC after PSM. Therefore, surgeons should be cautious when patients were with concurrent CRC and T2DM.

\section{Funding}

This study was supported by Chongqing Key Diseases Research and Application Demonstration Program (Colorectal Cancer Prevention and Treatment Technology Research and Application Demonstration [No. 2019ZX003]).

\section{Disclosure}

The authors report no conflicts of interest in this work.

\section{References}

1. Rawla P, Sunkara T, Barsouk A. Epidemiology of colorectal cancer: incidence, mortality, survival, and risk factors. Prz Gastroenterol. 2019;14(2):89-103. doi:10.5114/pg.2018.81072

2. Dekker E, Tanis PJ, Vleugels JLA, Kasi PM, Wallace MB. Colorectal cancer. Lancet. 2019;394(10207):1467-1480. doi:10.1016/S01406736(19)32319-0

3. Arnold M, Sierra MS, Laversanne M, et al. Global patterns and trends in colorectal cancer incidence and mortality. Gut. 2017;66(4):683-691. doi:10.1136/gutjnl-2015-310912

4. Cheng YX, Tao W, Zhang H, Peng D, Wei ZQ. Does liver cirrhosis affect the surgical outcome of primary colorectal cancer surgery? A meta-analysis. World J Surg Oncol. 2021;19(1):167. doi:10.1186/s12957021-02267-6 
5. Watanabe T, Momosaki R, Suzuki S, Abo M. Preoperative rehabilitation for patients undergoing colorectal cancer surgery: a retrospective cohort study. Support Care Cancer. 2020;28(5):2293-2297. doi:10.1007/s00520-019-05061-z

6. Li Y, Yan Y, Dai H, et al. Mastery of type 2 diabetes prevention and treatment knowledge by general practitioners in Shanghai: a cross-sectional study. BMC Fam Pract. 2021;22(1):189. doi:10.1186/s12875-021-01538-1

7. Roy A, Sahoo J, Kamalanathan S, et al. Diabetes and pancreatic cancer: exploring the two-way traffic. World $J$ Gastroenterol. 2021;27(30):4939-4962. doi:10.3748/wjg.v27.i30.4939

8. Chatterjee S, Khunti K, Davies MJ. Type 2 diabetes. Lancet. 2017;389(10085):2239-2251. doi:10.1016/S0140-6736(17)30058-2

9. Klil-Drori AJ, Azoulay L, Pollak MN. Cancer, obesity, diabetes, and antidiabetic drugs: is the fog clearing? Nat Rev Clin Oncol. 2017;14 (2):85-99. doi:10.1038/nrclinonc. 2016.120

10. Abudawood M. Diabetes and cancer: a comprehensive review. $J$ Res Med Sci. 2019;24:94. doi:10.4103/jrms.JRMS_242_19

11. Giovannucci E, Harlan DM, Archer MC, et al. Diabetes and cancer: a consensus report. Diabetes Care. 2010;33(7):1674-1685. doi:10.2337/dc10-0666

12. Guraya SY. Association of type 2 diabetes mellitus and the risk of colorectal cancer: a meta-analysis and systematic review. World $J$ Gastroenterol. 2015;21(19):6026-6031. doi:10.3748/wjg.v21. i19.6026

13. Zhu B, Wu X, Wu B, et al. The relationship between diabetes and colorectal cancer prognosis: a meta-analysis based on the cohort studies. PLoS One. 2017;12(4):e0176068. doi:10.1371/journal. pone. 0176068

14. Petrelli F, Ghidini M, Rausa E, et al. Survival of colorectal cancer patients with diabetes mellitus: a meta-analysis. Can J Diabetes. 2021;45(2):186-197.e2. doi:10.1016/j.jcjd.2020.06.009

15. Weiser MR. AJCC 8th edition: colorectal cancer. Ann Surg Oncol. 2018;25(6):1454-1455. doi:10.1245/s10434-018-6462-1

16. Clavien PA, Barkun J, de Oliveira ML, et al. The Clavien-Dindo classification of surgical complications: five-year experience. Ann Surg. 2009;250(2):187-196. doi:10.1097/SLA.0b013e3181b13ca2

17. Xu B, Zhou X, Li X, Liu C, Yang C. Diabetes mellitus carries a risk of esophageal cancer: a meta-analysis. Medicine. 2017;96(35):e7944. doi:10.1097/MD.0000000000007944

18. Peng D, Cheng YX, Zhang W. Does Roux-en-Y construction really bring benefit of type 2 diabetes mellitus remission after gastrectomy in patients with gastric cancer? A systematic review and meta-analysis. Diabetes Ther. 2020;11(12):2863-2872. doi:10.1007/ s13300-020-00934-7

19. Mills KT, Bellows CF, Hoffman AE, Kelly TN, Gagliardi G. Diabetes mellitus and colorectal cancer prognosis: a meta-analysis. Dis Colon Rectum. 2013;56(11):1304-1319. doi:10.1097/ DCR.0b013e3182a479f9

20. Jullumstrø E, Kollind M, Lydersen S, Edna TH. Diabetes mellitus and outcomes of colorectal cancer. Acta Oncol. 2009;48(3):361-367. doi:10.1080/02841860802637765
21. Amshoff Y, Maskarinec G, Shvetsov YB, et al. Type 2 diabetes and colorectal cancer survival: the multiethnic cohort. Int $J$ Cancer. 2018;143(2):263-268. doi:10.1002/ijc.31311

22. Ahmadi A, Noroozi M, Pourhoseingholi MA, et al. Effect of metabolic syndrome and its components on survival in colorectal cancer: a prospective study. J Renal Inj Prev. 2015;4(1):15-19. doi:10.12861/ jrip. 2015.05

23. Brown JC, Zhang S, Ou FS, et al. Diabetes and clinical outcome in patients with metastatic colorectal cancer: CALGB 80405 (alliance). JNCI Cancer Spectr. 2019;4(1):pkz078. doi:10.1093/ jncics/pkz078

24. Ma YS, Yang IP, Tsai HL, et al. High glucose modulates antiproliferative effect and cytotoxicity of 5-fluorouracil in human colon cancer cells. DNA Cell Biol. 2014;33(2):64-72. doi:10.1089/ dna.2013.2161

25. Saito A, Kitayama J, Horie H, et al. Metformin changes the immune microenvironment of colorectal cancer in patients with type 2 diabetes mellitus. Cancer Sci. 2020;111(11):4012-4020. doi:10.1111/ cas. 14615

26. Cheng HC, Chang TK, Su WC, Tsai HL, Wang JY. Narrative review of the influence of diabetes mellitus and hyperglycemia on colorectal cancer risk and oncological outcomes. Transl Oncol. 2021;14 (7):101089. doi:10.1016/j.tranon.2021.101089

27. Lonjon G, Boutron I, Trinquart L, et al. Comparison of treatment effect estimates from prospective nonrandomized studies with propensity score analysis and randomized controlled trials of surgical procedures. Ann Surg. 2014;259(1):18-25. doi:10.1097/ SLA.0000000000000256

28. Yap R, Wilkins S, Staples M, Oliva K, McMurrick PJ. The effect of diabetes on the perioperative outcomes of colorectal cancer surgery patients. PLoS One. 2016;11(12):e0167271. doi:10.1371/journal. pone. 0167271

29. Tan DJH, Yaow CYL, Mok HT, et al. The influence of diabetes on postoperative complications following colorectal surgery. Tech Coloproctol. 2021;25(3):267-278. doi:10.1007/s10151-020-02373-9

30. Kido H, Kato S, Funahashi K, et al. The metabolic parameters based on volume in PET/CT are associated with clinicopathological $\mathrm{N}$ stage of colorectal cancer and can predict prognosis. EJNMMI Res. 2021;11(1):87. doi:10.1186/s13550-021-00831-5

31. Peng D, Liu XY, Cheng YX, Tao W, Cheng Y. Improvement of diabetes mellitus after colorectal cancer surgery: a Retrospective Study of Predictive factors for type 2 diabetes mellitus remission and overall survival. Front Oncol. 2021;11:694997. doi:10.3389/ fonc.2021.694997

32. Pang QY, An R, Liu HL. Perioperative transfusion and the prognosis of colorectal cancer surgery: a systematic review and meta-analysis. World J Surg Oncol. 2019;17(1):7. doi:10.1186/s12957-018-1551-y

\section{Publish your work in this journal}

Cancer Management and Research is an international, peer-reviewed open access journal focusing on cancer research and the optimal use of preventative and integrated treatment interventions to achieve improved outcomes, enhanced survival and quality of life for the cancer patient.
The manuscript management system is completely online and includes a very quick and fair peer-review system, which is all easy to use. Visit http://www.dovepress.com/testimonials.php to read real quotes from published authors. 